\title{
Technical note: Determination of corn hardness in diverse corn germplasm using near-infrared reflectance baseline shift as a measure of grinding resistance
}

\author{
P. C. Hoffman, ${ }^{* 1}$ D. Ngonyamo-Majee, $\dagger$ and R. D. Shaver* \\ *Department of Dairy Science, University of Wisconsin, Madison 53706 \\ †Monsanto Company, Biotechnology Group, Mystic, CT 06355
}

\section{ABSTRACT}

Vitreousness and Stenvert kernel hardness characteristics in corn germplasm have been related to in situ and in vivo starch digestibility in lactating dairy cows. Because of the tedious nature of determining vitreousness by manual dissection or conducting Stenvert hardness measurements, it is challenging to screen corn germplasm for animal performance potential. The resistance of corn to grinding is typically measured in a Stenvert mill and quantified by grinding time and column height. The baseline shift (BLS) of near-infrared reflectance spectroscopy, which is a normal artifact of grinding-induced particle size differentiation, was calculated and evaluated as an alternative measure of grinding resistance. Stenvert grind time, column height, BLS, and ruminal DM degradability (RDMD) of 31 diverse corn germplasm harvested in triplicate at 2 maturities were made. After grinding, total and 6 partial $(100 \mathrm{~nm})$ BLS were calculated $\left[\Sigma \mathrm{x}_{\mathrm{i}}+\mathrm{x}_{\mathrm{j}} \ldots\right.$, where $\mathrm{x}_{\mathrm{i}}$ $=\log _{10}(1 / \mathrm{R})$ at the ith nanometer and $\mathrm{R}=$ reflectance] and compared with vitreousness, Stenvert grinding time, column height, or RDMD of corns using correlation and regression procedures. Total and all partial BLS were correlated with vitreousness, Stenvert measures, and RDMD. Relationships between BLS and vitreousness, Stenvert measures, or RDMD were stronger for partial BLS at shorter near-infrared wavelengths $(1,080-1,180$ $\mathrm{nm})$ than at longer wavelengths (i.e., $2,280-2,380 \mathrm{~nm}$ ) or total near-infrared BLS $(1,000-2,498 \mathrm{~nm})$. The partial BLS from 1,080 to $1,180 \mathrm{~nm}$ and vitreousness were better related to RDMD of corn germplasm than Stenvert grind time or column height.

Key words: near-infrared, corn, endosperm

Corns with greater percentage of vitreous endosperm have reduced in vitro or in situ DM degradation po-

\footnotetext{
Received August 17, 2009.

Accepted January 4, 2010.

${ }^{1}$ Corresponding author: pchoffma@wisc.edu
}

tential (Philippeau et al., 2000; Correa et al., 2002; Ngonyamo-Majee et al., 2008b). Trials conducted by Taylor and Allen (2005) and Lopes et al. (2009) demonstrated improved starch digestion in lactating dairy cows that were fed dry corns containing less vitreous endosperm.

Vitreousness of corn can be quantified in whole corn kernels by manual dissection (Correa et al., 2002). Corn kernels are soaked in water, the pericarp and germ are removed with a scalpel, and the floury (white, opaque) endosperm is separated from vitreous (yellow, glassy) endosperm with a scalpel. The weight of the vitreous endosperm is weighed and expressed as a percentage of the total endosperm.

The measurement of vitreousness has several shortcomings, including lack of random distribution in corn, manual dexterity requirements, and the fact that assay can be performed only on whole intact kernels of corn. As a result, numerous alternative methods have been evaluated to quantify differences of corn endosperm vitreousness or hardness. Fox and Manley (2009) reviewed alternative laboratory methods to access corn endosperm hardness, including the Stenvert hardness test (SHT). The SHT, originally developed to assess wheat hardness (Stenvert, 1974), has been used successfully to determined corn endosperm hardness (Pomeranz et al., 1985), and SHT measures in corn have been shown to be negatively related to ruminal DM degradability (Ngonyamo-Majee et al., 2008b). The SHT measures grinding resistance as time required to fill a $17-\mathrm{mL}$ volume, thereby relating hardness to grinding time. In addition, the total volume (column height) of a $20-\mathrm{g}$ sample is measured to assess the bulk density of the ground corn sample, associating lower column heights with increased hardness.

In reviewing the recent work of Ngonyamo-Majee et al. (2008a), who explored near-infrared reflectance spectroscopy (NIRS) equations to predict SHT grinding time and column height, an anomaly was noticed in the procedure. Ngonyamo-Majee et al. (2008a) developed and evaluated traditional chemometric NIRS 
prediction equations for SHT grinding time and column height using derivatives of NIRS spectra for 47 diverse corn germplasm. A fundamental of near-infrared light physics may have been overlooked in the report. The scatter of near-infrared light is a result of reflection, refraction, and random diffraction created by surface particles (Birth and Hecht, 1987). As particle size is reduced, the light-scattering interface is increased, resulting in increased light reflection (Birth and Hecht, 1987). More simply, the finer a given plant material is ground, the more light will be reflected (lower absorbance) because there are more particles to reflect light. In NIRS applications, variance in light reflection induced by particle size differentials in the sample is referred to as baseline shift (BLS; Hruschka, 1987), and chemometric applications (Hruschka, 1987) of NIRS are designed to mitigate or eliminate BLS by using derivatives of the spectra in lieu of absolute reflectance or absorbance. The grinding of plant material, with diverse physical texture differences, through the same grinding mill is known to result in particle size differentiation in the ground sample and results in BLS (Williams, 1987). The objective of this study was to assess whether the intrinsic BLS in NIRS could serve as an alternative measure of grinding resistance and whether BLS is related to ruminal DM degradability (RDMD) of corn.

The original data of 33 diverse corn germplasm evaluated by Ngonyamo-Majee et al. (2008b) were reevaluated, including a reevaluation of the NIRS spectra. Complete details of the corn germplasm, measures of vitreousness, kernel density, SHT, and RDMD procedures are described in detail by Ngonyamo-Majee et al. (2008a,b) and are not repeated in detail here. A brief overview of the project is as follows. Thirty-three diverse corn endosperms were grown at the University of Wisconsin-Madison and included a broad range of corn endosperm types. Endosperm types included opaque-2 (o2), sugary (su2), densely packed flint types, and a range of intermediates. Corns were planted in triplicate plots and harvested at 1/2 milkline and black-layer maturity stages. Vitreousness of corn endosperm types at both stages of maturity were determined by manual dissection. Corns were dried for $72 \mathrm{~h}$ at $40^{\circ} \mathrm{C}$ and hardness was determined by grinding a 20 -g sample using a Stenvert micro hammer cutter (Stenvert, 1974) mill fit with a 2 -mm screen operating at 3,600 rpm. Grind time required to fill a $17-\mathrm{mL}$ receptacle and total column height achieved after complete grinding of the $20 \mathrm{-g}$ sample was recorded. A subsample of corn kernels from each replicate of each corn germplasm at each maturity was ground through a Wiley mill (Arthur A. Thomas Co., Philadelphia, PA) fit with a $6-\mathrm{mm}$ screen and evaluated for RDMD. In situ RDMD was determined by incubating 8 replicate Dacron bags $(50 \mu \mathrm{m} ; 5 \times 5$ $\mathrm{cm})$ containing $1.5 \mathrm{~g}$ of corn/bag for 0 and $14 \mathrm{~h}$ in 2 cannulated steers.

Near-infrared reflectance spectra $(\mathrm{n}=198)$ were collected on all replicates $(\mathrm{n}=3)$ of the 33 corn germplasm at 2 maturity stages. Dried kernels were ground using a Stenvert micro hammer cutter mill fitted with a 1-mm screen and scanned in duplicate with a model 6500 near-infrared reflectance spectrophotometer (Foss NIRSystems, Laurel, MD). Spectral data were collected and saved at $2-\mathrm{nm}$ wavelength intervals as absorbance $\left[\log _{10}(1 / R)\right.$, where $R$ is the percentage reflectance].

To calculate BLS, spectral data in 2-nm increments from 1,000 to 2,498 $\mathrm{nm}$ were converted to an Excel 2007 (Microsoft Corp., Redmond, WA) format. One su2 inbred and 1 su2 cross germplasm were removed from the evaluation because su2 endosperm types are not typically grown for livestock production purposes. Total (1,000-2,498 nm) and 6 partial $(100 \mathrm{~nm})$ BLS were calculated for the remaining 31 corn germplasm as $\Sigma \mathrm{x}_{\mathrm{i}}+\mathrm{x}_{\mathrm{j}} \ldots$, where $\mathrm{x}_{\mathrm{i}}=\log _{10}(1 / \mathrm{R})$ at the ith nanometer and $\mathrm{R}=$ reflectance. The partial BLS were collected at 1,080 to $1,180,1,450$ to $1,550,1,700$ to $1,800,1,920$ to $2,020,2,060$ to 2,160 , and 2,280 to $2,380 \mathrm{~nm}$. Partial BLS was calculated using $100-\mathrm{nm}$ ranges to minimize the potential influence of a narrow absorptive region in the spectra. Partial BLS were not selected randomly but rather were selected on the following criteria. Partial BLS regions 1,450 to $1,550,1,700$ to $1,800,1,920$ to $2,020,2,060$ to 2,160 , and 2,280 to $2,380 \mathrm{~nm}$ were selected because these regions in the near-infrared spectra represented the widest ranges in BLS. Partial BLS region 1,080 to $1,180 \mathrm{~nm}$ was selected using the spectral correlation procedures of Shenk and Westerhaus (1991). The total and 6 partial (100 nm) BLS were compared with vitreousness, Stenvert grinding time, and column height or RDMD of corns using correlation and regression procedures of SAS (2001).

Complete summary statistics of vitreousness, kernel density, Stenvert measures, and RDMD of the corn germplasm are detailed in Ngonyamo-Majee et al. (2008a,b), but a brief review including BLS is presented in Table 1. Average vitreousness of corn germplasm endosperms was $69.0 \%$, with a 95 percentage unit range. The variation in vitreousness transcended into differences in grinding resistance as measured by Stenvert grinding time and column height. Grinding time to fill a $17-\mathrm{mL}$ column ranged from 9.5 to $31.5 \mathrm{~s}$ and column height achieved after grinding $20 \mathrm{~g}$ ranged from 6.3 to $8.7 \mathrm{~cm}$. Likewise, 14-h in situ RDMD of the 6-mm ground corn germplasm ranged approximately 20 percentage units. 
Table 1. Summary statistics of vitreousness, Stenvert hardness, near-infrared reflectance spectroscopy baseline shift (NIRS BLS), and in situ DM degradability of 31 diverse corn germplasms

\begin{tabular}{|c|c|c|c|c|c|}
\hline Item & Mean & Minimum & Maximum & SD & $\mathrm{RMR}^{1}$ \\
\hline \multicolumn{6}{|l|}{ Endosperm } \\
\hline Vitreousness, $\%$ of endosperm & 69.0 & 0.0 & 95.0 & 24.0 & 1.38 \\
\hline Stenvert grinding time, $\mathrm{s}$ & 20.33 & 9.50 & 31.50 & 3.92 & 1.08 \\
\hline Stenvert column height, $\mathrm{cm}$ & 7.27 & 6.30 & 8.70 & 0.55 & 0.33 \\
\hline \multicolumn{6}{|l|}{ NIRS BLS, nm } \\
\hline $1,080-1,180$ & 4.86 & 3.00 & 6.99 & 0.81 & 0.82 \\
\hline $1,450-1,550$ & 18.42 & 12.67 & 28.40 & 2.92 & 0.85 \\
\hline $1,700-1,800$ & 16.91 & 11.23 & 26.09 & 2.82 & 0.88 \\
\hline $1.920-2,020$ & 23.52 & 16.82 & 36.48 & 3.64 & 0.84 \\
\hline $2,060-2,160$ & 26.80 & 19.90 & 40.79 & 3.87 & 0.78 \\
\hline $2,280-2,380$ & 29.31 & 21.76 & 44.60 & 4.25 & 0.78 \\
\hline $1,000-2,498$ & 265.48 & 187.30 & 404.48 & 40.53 & 0.82 \\
\hline \multicolumn{6}{|l|}{ In situ } \\
\hline 0-h DM loss, $\%$ of DM & 9.40 & 0.00 & 19.60 & 2.77 & 2.09 \\
\hline 14-h DM degradation, $\%$ of DM & 34.96 & 24.30 & 55.30 & 5.96 & 0.89 \\
\hline
\end{tabular}

${ }^{1}$ Range:mean ratio.

The BLS of the corn germplasm are also presented in Table 1. The highest BLS was observed for total near-infrared BLS (1,000-2,498 nm) simply because the sum of $\log _{10}(1 / R)$ is constructed from 749 data points as compared with 50 data points for the 100-nm partial BLS calculations. Partial BLS numerical values increase when BLS is calculated from longer 100-nm near-infrared wavelength segments, which is an intrinsic aspect of near-infrared reflectance physics. As wavelengths in the near-infrared region increase, reflectance generally decreases and absorption correspondingly increases (Birth and Hecht, 1987). Because $\log _{10}(1 / \mathrm{R})$ is an expression of absorption, it is logical that the sum values of partial BLS increase when partial BLS is calculated at longer wavelength in the near-infrared region as compared with shorter wavelength regions (i.e., $2,280-2,380$ vs. $1,080-1,180 \mathrm{~nm}$ ). Because the values of total and partial BLS cannot be readily compared, a simple range:mean ratio (RMR) of BLS was calculated in an effort to express total and partial BLS in similar terms and is presented in Table 1. The RMR of total and all partial BLS is near 0.84, indicating that the mean range relationship of any of the BLS calculated is relatively similar. The RMR is slightly higher when partial BLS is calculated at shorter near-infrared wavelengths, indicating that measurement sensitivity within the BLS is perhaps more efficient at shorter wavelength segments even though the absolute BLS are smaller when calculated from shorter near-infrared wavelength regions.

Pearson correlations between vitreousness, Stenvert grinding time, column height, RDMD, and BLS are presented in Table 2. Total $(1,000-2,498 \mathrm{~nm})$ and all partial BLS for the corn germplasm were positively correlated $(P<0.001)$ with vitreousness and Stenvert grinding time and negatively correlated with Stenvert column height. As vitreousness or Stenvert grinding time increased, BLS increased. The dynamics of the relationship between BLS (1,080-1,180 nm) and Stenvert grinding time are demonstratively presented in Figure 1. The notable decrease in absorbance $\left[\log _{10}(1 / R)\right]$ at $1,100 \mathrm{~nm}$ for all corns is a NIRS nuance as the spectrophotometer shifts between the visible and near-infrared regions.

Because plotting all 198 NIRS spectra is cumbersome, the mean replicate $\times$ maturity absorbance at 1,080 to 1,180 nm of Oh43 corn germplasm and 4 nearisogenic variants of Oh43 carrying the o2, floury-2 (fl2), amylase-extentder-1 (ae1), and soft endosperm (h1) alleles were plotted. The NIRS absorbance at 1,080 to $1,180 \mathrm{~nm}$ and Stenvert grinding times of Oh43 and ae1 Oh43 are notably higher than NIRS absorbance and Stenvert grinding times of the softer endosperm (Ngonyamo-Majee et al. (2008a,b), o2, fl2, and $h 1 \mathrm{Oh} 43$ variants. Because Oh43 or ae1 Oh43 are more vitreous endosperms and more resistant to grinding, the NIRS baseline is shifted up as compared with the less vitreous fl2, o2, and $h 1$ endosperm types (Robutti et al., 2000; Ngonyamo-Majee et al. 2008a,b).

All BLS were significantly correlated with vitreousness, and SHT measures correlations were stronger for partial BLS calculated from shorter near-infrared wavelength regions as compared with longer wavelength regions. The correlations between vitreousness $(\mathrm{r}=0.72)$, Stenvert grinding time $(\mathrm{r}=0.53)$, and Stenvert column height $(\mathrm{r}=-0.65)$ were maximal when compared with partial BLS at 1,080 to $1,180 \mathrm{~nm}$. It is unclear why BLS at shorter near-infrared wavelength regions is better related to SHT measures, but it may be related to the lack of interference of primary organic bonds associ- 


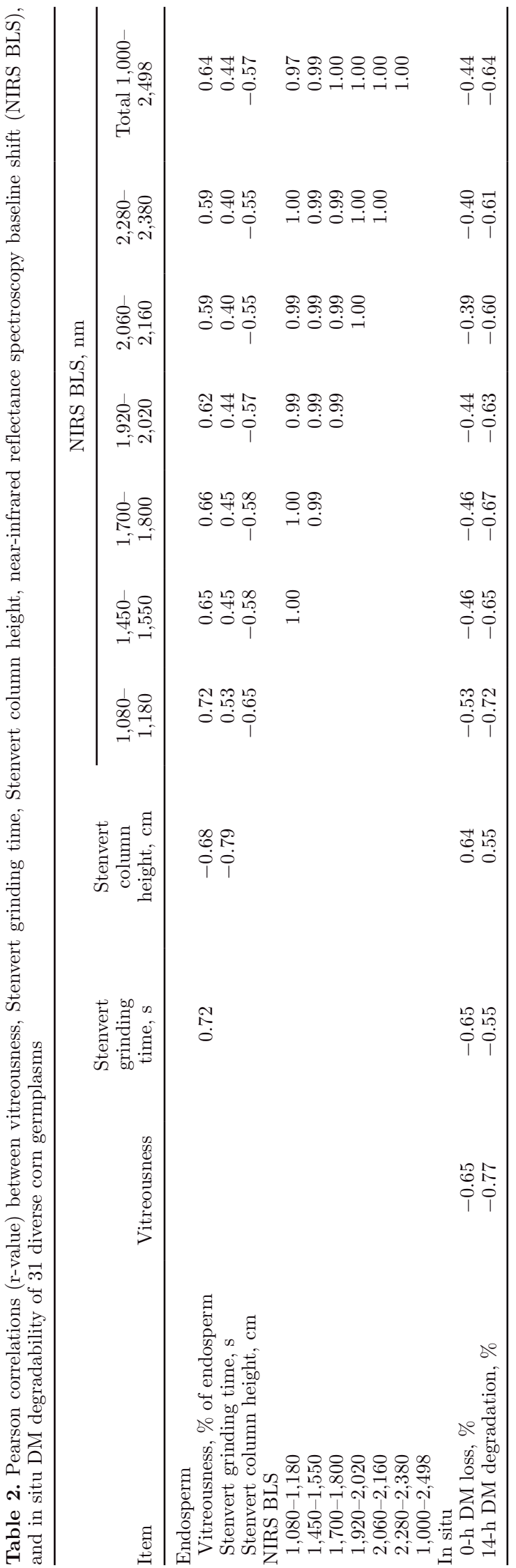

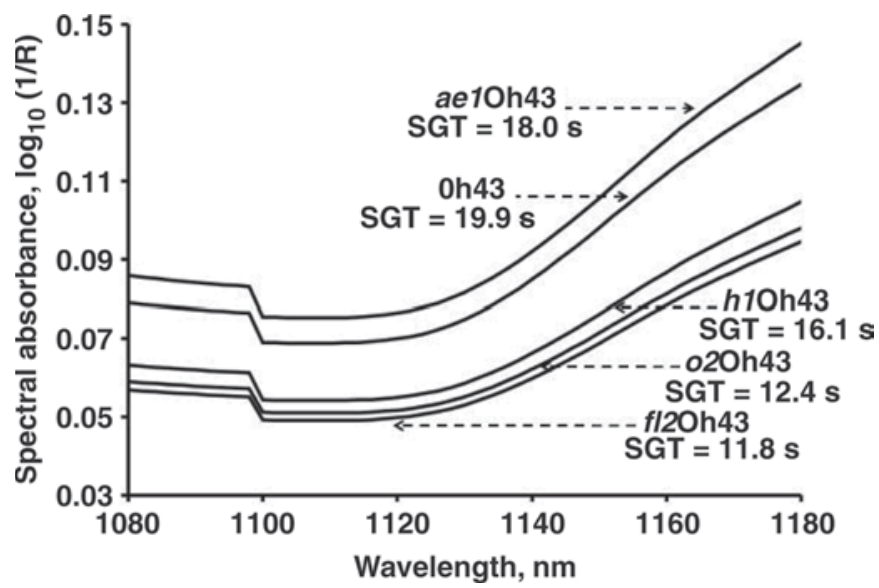

Figure 1. Spectral absorbance $\left[\log _{10}(1 / R)\right.$, where $R=$ reflectance $]$ at 1,080 to $1,180 \mathrm{~nm}$ for $\mathrm{Oh} 43$ and 4 near-isogenic variants of Oh43 carrying opaque-2 (o2), floury-2 (fl2), amylase-extentder-1 (ae1), or soft endosperm $(h 1)$ alleles. The vitreousness of Oh43 and o2, fl2, $a e 1$, and $h 1$ variants was $58.8,-12.5,2.5,41.5$, and $7.4 \%$ of the endosperm, respectively. Mean maturity $\times$ replication Stenvert grinding times (SGT) as measured by Ngonyamo-Majee et al. (2008a, 2008b) are also defined for each germplasm.

ated with protein, water, and starch, which are most commonly associated with near-infrared wavelengths $>1,400 \mathrm{~nm}$ (Hruschka, 1987).

Correlations between vitreousness, SHT measures, BLS, and RDMD are presented in Table 2. Total $(1,000-2,498 \mathrm{~nm})$ and all partial BLS for the corn germplasm were negatively correlated $(P<0.001)$ with 14-h in situ RDMD, with the most defining negative correlation $(\mathrm{r}=-0.72)$ occurring for the partial BLS at 1,080 to $1,180 \mathrm{~nm}$. The partial BLS at 1,080 to $1,180 \mathrm{~nm}$ was better correlated to 14 -h in situ RDMD than Stenvert grinding time or column height and was equally correlated with RDMD as vitreousness.

The practical implications of this technical note are subtle. For research and education programs wishing to evaluate corn germplasm for chemical composition (CP, starch, fat, and so on) and physical texture (hardness, vitreousness), multiple laboratory techniques may not be necessary. Traditionally, corn samples are dried, ground, and scanned on NIRS instruments to rapidly determine chemical composition (i.e., CP, starch, fat) using chemometric-derived equations. Data from this project suggest that using the same spectra used for predicting chemical composition and calculating BLS from 1,080 to $1,180 \mathrm{~nm}$ may be equally as informative in regard to RDMD as Stenvert measures or vitreousness.

However, some interpretive caution of this technical note is advised. All SHT and BLS data in this study were derived from grinding diverse corn germplasm in a Stenvert micro hammer cutter mill. This was required 
to make direct comparisons between SHT and BLS, but more commonly corn samples are prepared for NIRS scanning by grinding corn in Udy (Udy Corp., Boulder, CO) or Wiley (Arthur H. Thomas, Philadelphia, PA) mills fit with 1-mm screens. However, abundant archive data (Williams, 1987) suggest that the physical texture or hardness of the plant material influences particle size distribution when samples are ground using the aforementioned mill types. Likewise, it is well known (Williams, 1987) that switching mill types, or screen aperture size, or both, will directly influence BLS. Therefore, maintaining continuity in mill type and screen size would be a critical condition associated with this NIRS application.

\section{ACKNOWLEDGMENTS}

The authors thank Pat Flannery (Department of Agronomy, University of Wisconsin-Madison) and Lisa Bauman (Department of Soil Science, University of Wisconsin-Madison) for their generous technical assistance with this project.

\section{REFERENCES}

Birth, G. S., and H. G. Hecht. 1987. The physics of near-infrared reflectance. Pages 1-16 in Near-Infrared Technology in the Agricultural and Food Industries. Am. Assoc. Cereal Chem., St Paul, MN.

Correa, C. E. S., R. D. Shaver, M. N. Pereira, J. G. Lauer, and K. Kohn. 2002. Relationship between corn vitreousness and ruminal in situ starch degradability. J. Dairy Sci. 85:3008-3012.
Fox, G., and M. Manley. 2009. Hardness methods for testing maize kernels. J. Agric. Food Chem. 57:5647-5657.

Hruschka, W. R. 1987. Data analysis: Wavelength selection methods. Pages 35-53 in Near-Infrared Technology in the Agricultural and Food Industries. Am. Assoc. Cereal Chem., St. Paul, MN.

Lopes, J. C., R. D. Shaver, P. C. Hoffman, M. S. Akins, and S. J. Bertics. 2009. Type of corn endosperm influences nutrient digestibility in lactating dairy cows. J. Dairy Sci. 92:4541-4548.

Ngonyamo-Majee, D., R. D. Shaver, J. G. Coors, D. Sapienza, and J. G. Lauer. 2008a. Relationship between kernel vitreousness and dry matter degradability for diverse corn germplasm. I. Development of near-infrared reflectance spectroscopy calibrations. Anim. Feed Sci. Technol. 142:247-258.

Ngonyamo-Majee, D., R. D. Shaver, J. G. Coors, D. Sapienza, and J. G. Lauer. 2008b. Relationship between kernel vitreousness and dry matter degradability for diverse corn germplasm. II. Ruminal and post-ruminal degradabilities. Anim. Feed Sci. Technol. 142:259274

Philippeau, C., J. Landry, and B. Michalet-Doreau. 2000. Influence of the protein distribution of maize endosperm on ruminal starch degradability. J. Sci. Food Agric. 80:404-408.

Pomeranz, Y., Z. Czuchajowska, C. R. Martin, and F. S. Lai. 1985. Determination of corn hardness by the Stenvert Hardness Test. Cereal Chem. 62:108-112.

Robutti, J., F. Borras, M. Ferrer, M. Percibaldi, and C. A. Knutson. 2000. Evaluation of quality factors in Argentine maize races. Cereal Chem. 77:24-26.

SAS. 2001. SAS User's Guide: Statistics. SAS Inst. Inc., Cary, NC.

Shenk, J. S., and M. O. Westerhaus. 1991. Infrasoft International Software for Near Infrared Instruments. NIR Systems Inc., Silver Spring, MD.

Stenvert, N. L. J. 1974. Grinding resistance, a simple measure of wheat hardness. Flour Anim. Feed Milling. 156:24-27.

Taylor, C. C., and M. S. Allen. 2005. Corn grain endosperm type and brown midrib 3 corn silage: Site of digestion and ruminal digestion kinetics in lactating cows. J. Dairy Sci. 88:1413-1424.

Williams, P. 1987. Variables affecting near-infrared reflectance spectroscopic analysis. Pages 143-148 in Near-Infrared Technology in the Agricultural and Food Industries. Am. Assoc. Cereal Chem., St. Paul, MN. 